\title{
SEMANTIC IMAGE RETRIEVAL USING RELEVANCE FEEDBACK
}

\author{
Pushpa B. Patil ${ }^{1}$ and Manesh Kokare ${ }^{2}$ \\ ${ }^{1}$ Dept. of Computer Engineering, BLDEA's, V.P.Dr. P. G. Halakatti CET. Bijapur, India \\ Pushpa_mserediffmail.com \\ ${ }^{2}$ Dept. of Electronic and Telecommunication, S.G.G.S, Institute of Engineering and \\ Technology, Nanded, India \\ mbkokare@yahoo.com
}

\begin{abstract}
This paper presents optimized interactive content-based image retrieval framework based on AdaBoost learning method. As we know relevance feedback $(R F)$ is online process, so we have optimized the learning process by considering the most positive image selection on each feedback iteration. To learn the system we have used AdaBoost. The main significances of our system are to address the small training sample and to reduce retrieval time. Experiments are conducted on 1000 semantic colour images from Corel database to demonstrate the effectiveness of the proposed framework. These experiments employed large image database and combined RCWFs and DT-CWT texture descriptors to represent content of the images.
\end{abstract}

\section{KEYWORDS}

Content-Based Image Retrieval (CBIR), Relevance Feedback (RF), Rotated Complex Wavelet Filters (RCWFs), Dual Tree Complex Wavelet (DT-CWT), and Image retrieval

\section{INTRODUCTION}

Due to advancement in digital technology, there is a rapid growth of digital information. Because there is a development of the internet and availability of digital devices such as scanners, digital cameras etc. This huge digital information arises the challenge of various digital search applications in several areas such as medicine, commerce, education, and crime prevention. To do these tasks CBIR was introduced in the early 1980. CBIR uses the low level features like colour, texture and shape to retrieve the most similar images stored in the database. With these low level features, user's perception on the images can not be achieved. Since different users perception is different on same images. It is the big disadvantage of the CBIR. To overcome this, relevance feedback (RF) was introduced into CBIR in 1998[5]. There is good review on CBIR [1-4]

$\mathrm{RF}$ is an online process, which tries to learn the user perception interactively; initially RF was designed for text-based information retrieval systems. Later it was introduced into CBIR during mid 1990's, with the involvement of the user in the retrieval loop to reduce the "semantic gap" between query representation (low level features) and user perception (high level concepts). RF has been proved to provide effective and efficient retrieval performance improvement in CBIR systems through interactive learning based on the user feedback [5-6].

DOI : 10.5121/ijwest.2011.2411 
International Journal of Web \& Semantic Technology (IJWesT) Vol.2, No.4, October 2011

\subsection{Related Work}

Recently, many researchers began to consider the RF as a classification or semantic learning problem. That is a user provides positive and/or negative examples, and the systems learn from such examples to separate all data into relevant and irrelevant groups. Hence many classical machine learning schemes may be applied to the RF, which include decision tree learning [7], Bayesian learning [8]-[9], support vector machines [10], boosting [11] and so on. There is good review on RF in [12]. The process of learning is very difficult task in RF [12]-[14], due to the following reasons

Firstly small training data, which makes difficult to apply most of the learning methods such as linear discriminate fisher classifier and relevance vector machine (RVM). Though the RVMs are sparser than the SVMs and use less number of kernel functions. Secondly training data is asymmetrical, which creates a too much imbalance between the relevant and irrelevant images.Finally, In RF, for every iteration we have to perform both training and testing online, which takes more real time.

For visual representation of the images, we employed the global texture features dual tree complex wavelet (DT-CWT) and rotated complex wavelet filters (DT-RCWF) presented in [18], which provides very efficient performance. Much of the work on RF uses the low-level representation using discrete wavelet transform (DWT) [15], Gabor filters [16] and co-occurrence matrix [19][20] for textures. In order to retrieve the general purpose images like artificial objects and natural scenes most of the time textural features are combined with colour and shape to get better retrieval performance. However, they still suffers from the poor directional sensitivity, shift variant and, redundancy. From these combined features we may get better retrieval performance but not efficient one because as we increase number of features it increases the dimensionality of feature space. With such high dimensional feature space, RF may become impractical for even medium sized databases [14]. In order to store and process these high dimensional feature vectors it requires more memory space and time. So, to make our system efficient, we have to consider two factors namely time complexity and space complexity together with better retrieval performance. To overcome above problem, we propose to use the new rotated complex wavelet filters which gives both better and efficient retrieval performance..

\subsection{Our Approach}

In this paper we have used our earlier recent work [18] to extract more compact effective low level features, to improve the retrieval performance in terms of speed, storage and accuracy by using the rotated complex wavelet filters and dual tree complex wavelet transform jointly. These combined features gives information in $\left\{\begin{array}{l}\left(+15^{\circ},+45^{\circ},+75^{\circ},-15^{\circ},-45^{\circ},-75^{\circ}\right), \\ \left.0^{\circ},+30^{\circ},+60^{\circ},+90^{\circ}, 120^{\circ},-30^{\circ}\right)\end{array}\right\} \quad$ twelve different orientations. Further, to reduce the significant gap between low level feature and high level concepts, we have proposed a new RF approach and it is tested using AdaBoost. We found that proposed RF framework provides efficient retrieval performance in very few feedback iterations. A new relevance feedback approach, which is based on ADABoost, uses the relevant and irrelevant examples. Our extensive experiments using proposed RF with AdaBoost on standard general purpose database show significant improvements with respect to retrieval performance

The rest of the paper is organized as follows. In section 2, we have, explained the concept of AdaBoost. In section 3, experimental results are discussed. Finally, the conclusion is given in section 4 . 


\section{ADABOOST}

AdaBoost was introduced in 1995 by Freund and Schapire [24] as an efficient algorithm of the ensemble learning field. It is used to boost the classification performances of a weak learner. It does this by combining a collection of weak classification functions to form a stronger classifier. AdaBoost combines iteratively the weak classifiers by taking into account a weight distribution on the training samples such that more weight is attributed to samples misclassified by the previous iterations.

Consider a two classification problem, in which the training data comprises input vectors $X_{1}, \ldots, X_{N}$ along with corresponding binary target variables $t_{1}, \ldots, t_{n}$, where $t_{n} \in\{-1,1\}$. each data point is given an associated weighting parameter $w_{n}$, which is initially set $1 / N$ for all data points. We shall suppose that we have a procedure available for training a base classifier using weighted data to give a function $y(x) \in\{-1,1\}$. At each stage of the algorithm, AdaBoost trains a new classifier using a data set in which the weighting coefficients are adjusted according to the misclassified data points. Finally, when the desired number of base classifiers has been trained, they are combined to form a committee using coefficients that give different weight to different base classifiers.

\subsection{Proposed Algorithm}

The following algorithm describes the proposed methodology. It optimizes the testing data by removing the irrelevant images from the database on every iteration. Hence it reduces the retrieval time.

Input: Query image. Begin

Output: Retrieved images.

Retrieval of top T images from the database using CBIR.

Repeat until user satisfaction or result remains same

Begin

User feedback (ie labelling of images as relevant or irrelevant).

Learn the labeled images with AdaBoost.

Remove the irrelevant images from DB.

Canberra distance between relevant images returned by the learning system and query Image.

Sort distance vector.

Display top T images.

End

End

\subsection{Image Retrieval}

To conduct the experiments, each image from database is decomposed using DT-CWT and DTRCWF up to third level and two different sets of features were computed as follows.

To construct the feature vectors of each image in the database, we decomposed each image using DT-CWT and DT-RCWF up to third level. The Energy and Standard Deviation (STD) were computed separately on each subband and the feature vector was formed using these two parameter values. The retrieval performance with combination of these two feature parameters 
always outperformed that using these features individually [18]. The Energy $\left(E_{k}\right)$ and $\left(\sigma_{k}\right)$ Standard Deviation of $k^{\text {th }}$ subband is computed as follows.

$$
\begin{gathered}
E_{k}=\frac{1}{M \times N} \sum_{i=1}^{M} \sum_{j=1}^{N}\left|W_{k}(i, j)\right| \\
\sigma_{k}=\left[\frac{1}{M \times N} \sum_{i=1}^{N} \sum_{j=1}^{M}\left(W_{k}(i, j)-\mu_{k}\right)^{2}\right]^{\frac{1}{2}}
\end{gathered}
$$

Where $W_{k}(i, j)$ is the $k^{\text {th }}$ wavelet-decomposed subband, $M \times N$ is the size of wavelet decomposed subband, and $\mu_{k}$ is the mean of the subband. The resulting feature vector using energy and standard deviation are $\bar{f}_{E}=\left[\begin{array}{llll}E_{1} & E_{2} & \ldots & E_{n}\end{array}\right] \quad$ and $\bar{f}_{\sigma}=\left[\begin{array}{llll}\sigma_{1} & \sigma_{2} & \ldots & \sigma_{n}\end{array}\right] \quad$ respectively. So combined feature vector is $\bar{f}_{\sigma E}=\left[\begin{array}{llllllll}\sigma_{1} & \sigma_{2} & \ldots & \sigma_{n} & E_{1} & E_{2} & \ldots & E_{n}\end{array}\right]$

\subsection{Image Matching}

We have randomly selected any one of the 1856 images as a query image from texture images. Query image is further processed to compute the feature vector as given in section 2.2. Canberra distance metric is used as a similarity measure. If $x$ and $y$ are the feature vectors of the database and query image respectively, and have dimension $d$, then the Canberra distance is given by

$$
\operatorname{Canb}(\mathrm{x}, \mathrm{y})=\sum_{i=1}^{d} \frac{\left|x_{i}-y_{i}\right|}{\left|x_{i}\right|+\left|y_{i}\right|}
$$

\section{EXPERIMENTS}

To evaluate the performance of a proposed system, we have used the Corel image database [25]. The experiments were conducted using MATLAB 7.0 with Intel core2Duo, 1 GB RAM machine

\subsection{Corel Image Database}

It contains 1000 colour photographs of resolution $384 \times 256$ pixels, covering a wide range of semantic categories, from natural scenes to artificial objects [25]. The database is partitioned into ten categories, each with 100 photographs.

\subsection{Performance Measures}

For experimental results, it is significant to define a suitable metric for performance evaluation. We have used Average precision and it is defined as

$$
\text { precision }=\frac{\text { relevant images retrived in top T returns }}{T}
$$


For experimental results, there are 10 categories of images and in each category 100 natural colour images. For testing we have selected randomly 5 images from each category as query images (altogether 50 images). The reported results of average precision are obtained by taking an average over the 50 queries.

For each experiment, one image was selected at random as the query image from each category and thus the retrieved images were obtained. Then, the users were asked to identify those images that are related to their expectations from the retrieved images. These selected images were used as feedback images for next iteration. Finally, we have computed the average accuracy of all the categories in the database. Each image category contains 100 images. The feedback processes were performed 5 times and number of returned images $\mathrm{T}$ is 20 .

Fig.1 describes detailed comparison of the average retrieval precision obtained using R. Ding[25] and AdaBoost on every feedback iteration of the randomly selected image from each category of texture database. The main observation of proposed RF using AdaBoost gives better retrieval performance comparing with R. Ding method. From the Fig. 1, we observed that, there is a rapid increase in retrieval performance with each feedback iteration of proposed RF using AdaBoost learning algorithm. Retrieval performance is improved from $57.2 \%$ to $92.50 \%$ from first iteration to the fifth iteration using AdaBoostRF

We illustrated these observations using graph in Fig. 1 and results are also tabulated in table 1.

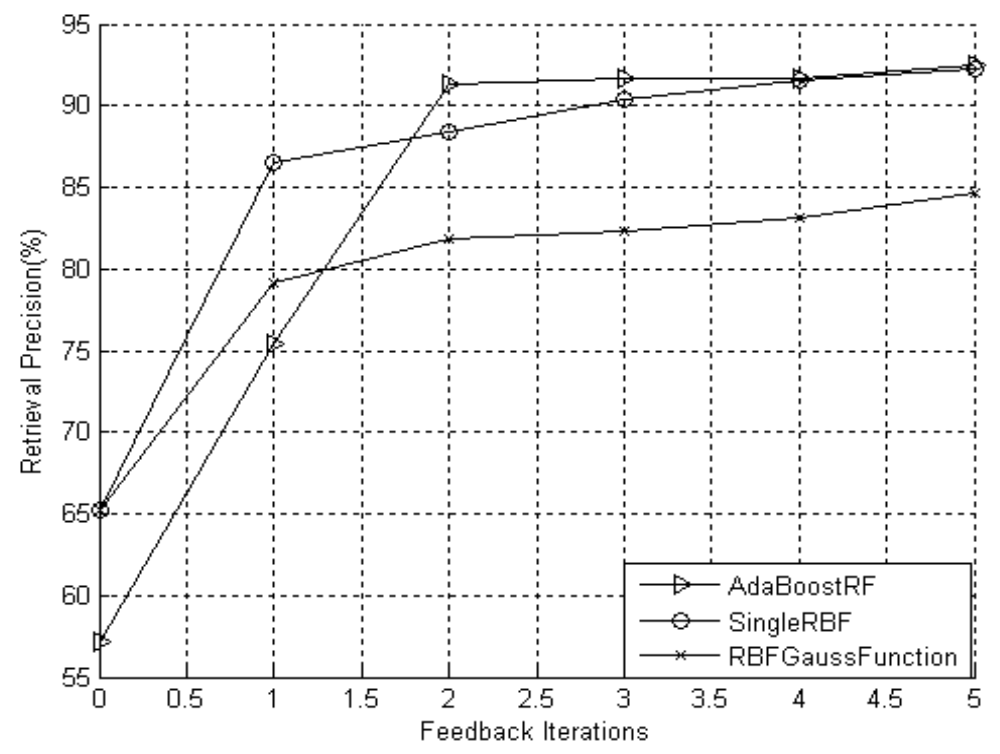

Figure 1. Average precision versus iteration curves for images

Table 1

Average retrieval Precision on each feedback iterations

\begin{tabular}{|l|l|l|l|l|l|l|}
\hline Approach & CBIR & $\begin{array}{l}1^{\text {st }} \\
\text { iteration }\end{array}$ & $\begin{array}{l}2^{\text {nd }} \\
\text { iteration }\end{array}$ & $\begin{array}{l}3^{\text {rd }} \\
\text { iteration }\end{array}$ & $\begin{array}{l}4^{\text {th }} \\
\text { iteration }\end{array}$ & $\begin{array}{l}5^{\text {th }} \\
\text { iteration }\end{array}$ \\
\hline AdaBoostRF & 57.2 & 75.4 & 91.32 & 91.70 & 91.70 & 92.5 \\
\hline Single_RBF & 65.2 & 86.5 & 88.4 & 90.4 & 91.5 & 92.3 \\
\hline RBFGaussFunction & 65.2 & 79.2 & 81.9 & 82.3 & 83.1 & 84.6 \\
\hline
\end{tabular}




\subsection{Image Retrieval Examples}

We use an example to illustrate the performance improvement of the proposed approach in Fig. 2(a)-2(e) for Corel database. Fig.2 (a) is the result of CBIR using combined features (RCWT+DT-CWT), in which among top 20 images, 8 images belongs to the desired(African people)category(i.e images 1-3 and image 5,12,14,15, and 17) and remaining 13 belongs to irrelevant category. So we got $40.0 \%$ retrieval precision from CBIR. Fig.2 (b)-(e) shows rapid performance improvement of the proposed approach using the AdaBoost for Corel database. From Fig. 2(b) to 2(e), we can observe that retrieval precision increasing from $80 \%$ to $100 \%$ from first iteration to fifth iteration of relevance feedback and it remains same in further iterations
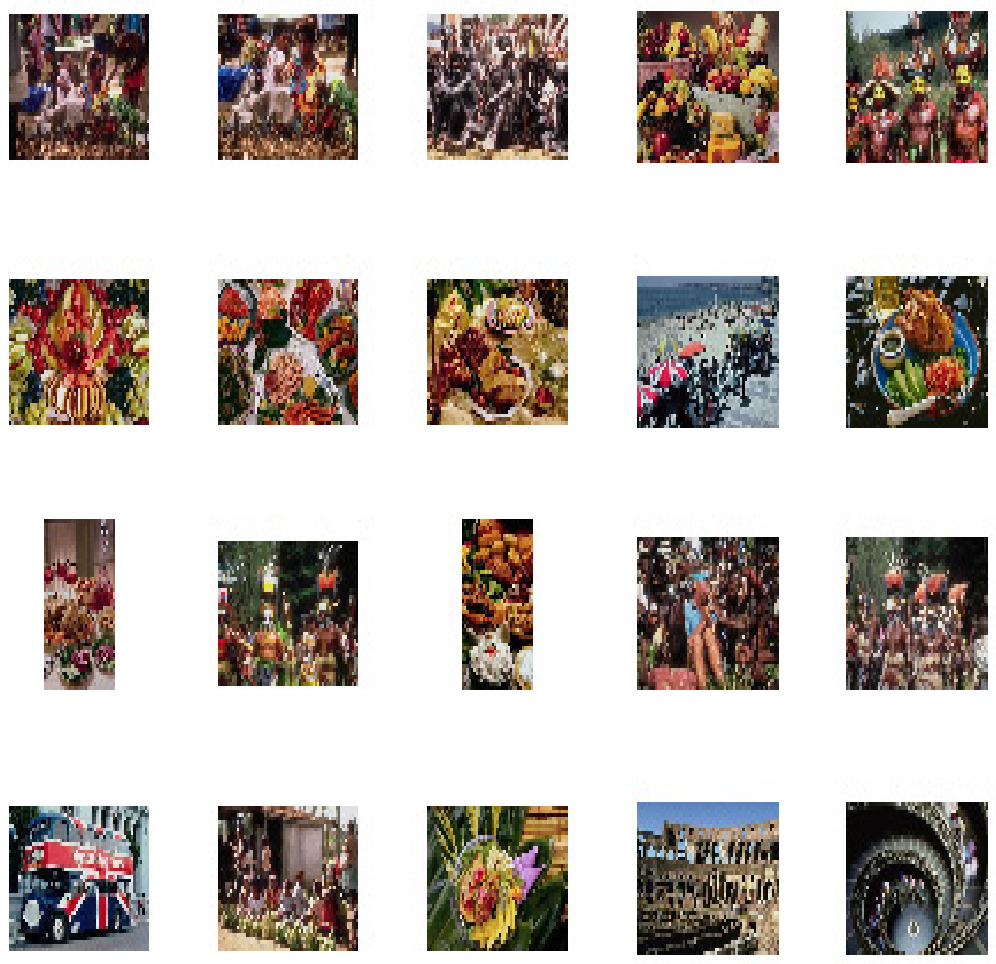

Fig 2(a) Result of CBIR using Combined features (RCWF +DT_CWT) (8/20) 


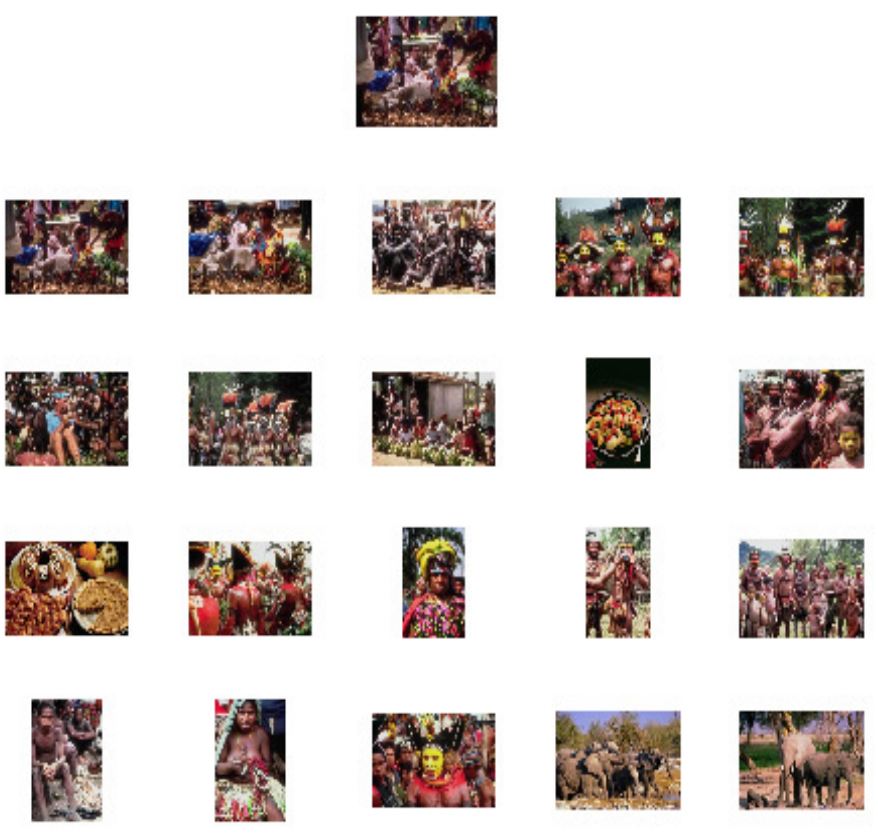

Fig. 2(b) Result after first feedback iteration using AdaBoostRF(16/20)
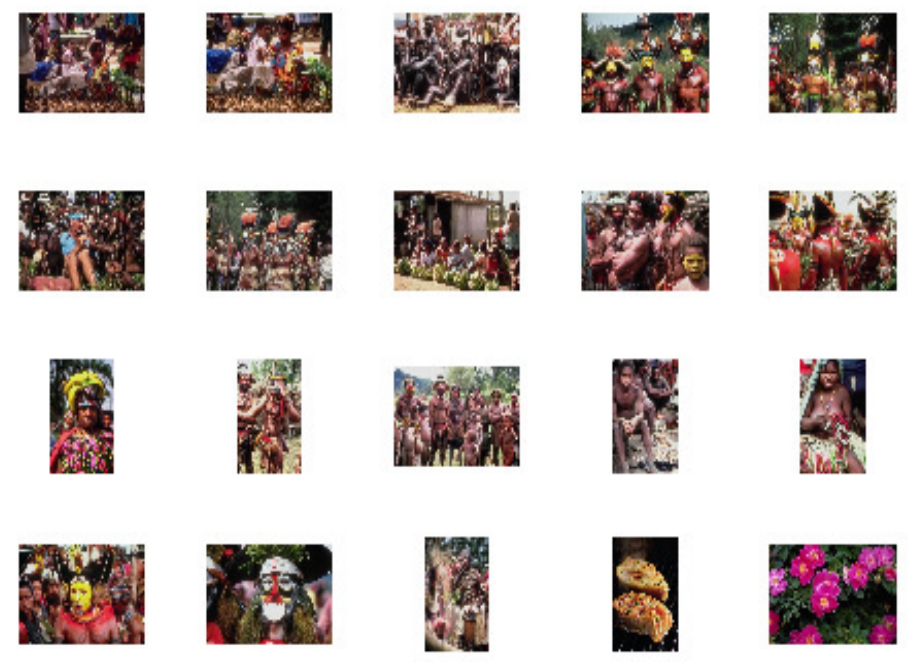

Fig.2(c) Result after Third feedback iteration using AdaBoostRF (18/20) 


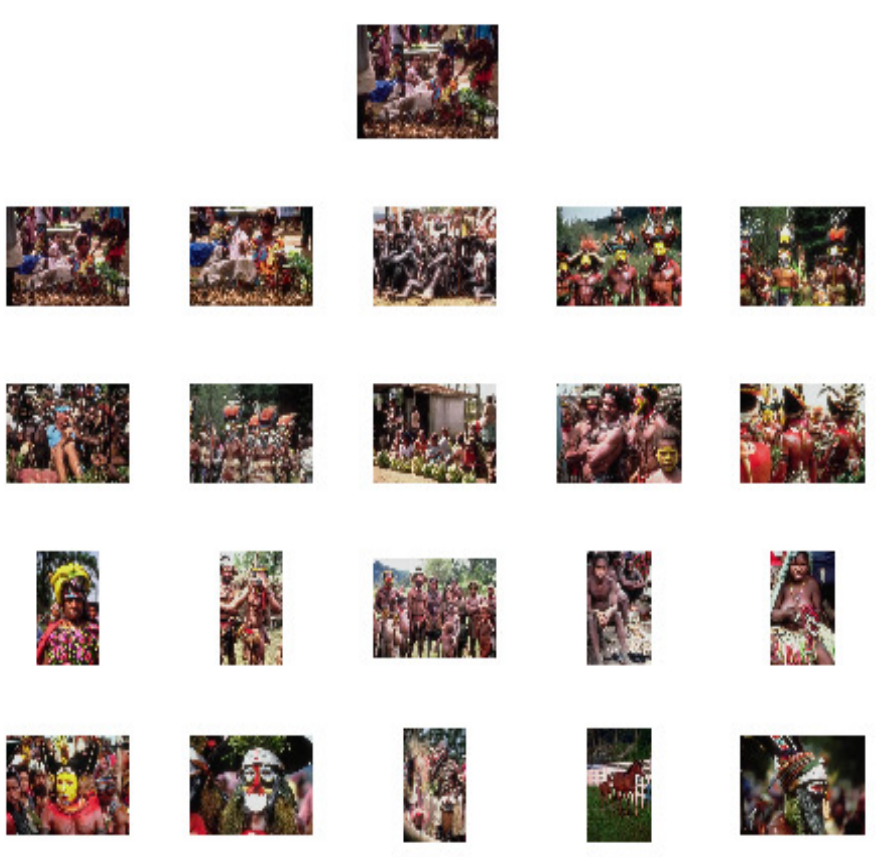

Fig.2 (d) Result after fourth feedback iteration using AdaBoostRF(19/20)

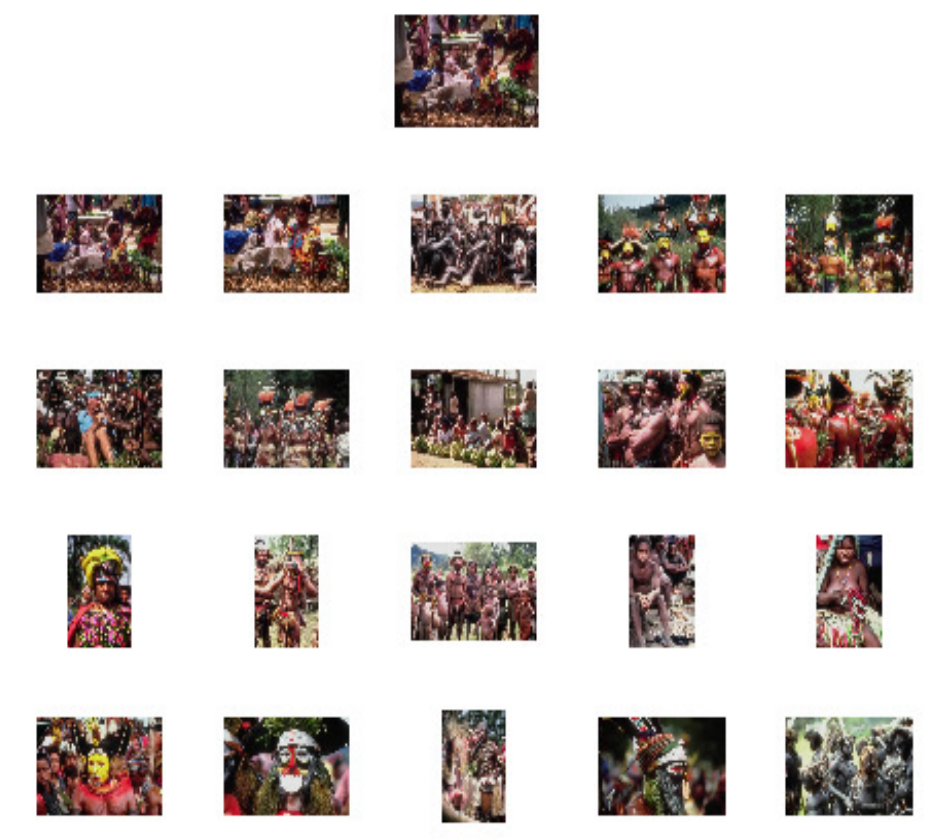

Fig.2 (e) Result after fifth feedback iteration using AdaBoostRF(20/20) 
International Journal of Web \& Semantic Technology (IJWesT) Vol.2, No.4, October 2011

\section{Conclusions}

In this paper, an active relevance feedback framework has been proposed to retrieve the semantic images. The proposed approach address the small training data problem in RF and optimizing the testing set in order to reduce the retrieval time. This relevance feedback framework is tested using AdaBoost with texture features. The RF framework is tested on large scale standard general purpose colour image database. The framework has demonstrated very promising retrieval accuracy. From experimental results, we found that RF using AdaBoost with combined texture features RCWF and DT-CWT gives better retrieval performance

\section{REFERENCES}

[1] Ritendra Datta, Dhiraj Joshi, Jia Li, and James Z. Wang. ,(2008), "Image Retrieval: Ideas, Influences, and Trends of the New Age," ACM Computing Surveys, Vol. 40, No. 2 article 5, pp.5:15:60.

[2] Rui, Y., Hung, T.S., Chang, S.F(1999), "Image retrieval: Current Techniques, Promising Directions and Open Issues,” J. Visual Comm. and Image Representation 10, pp. 39-62.

[3] Smeulders, A. W. M., Worring, M., Santini, S., Gupta, A., Jain R.(2000), "Content -based Image Retrieval at the End of the Early Years," IEEE trans. Pattern Anal. Machine Intell. 22(12), pp.13491380 .

[4] Kokare, M., Chatterji, B. N., Biswas P. K., (2002)“A Survey on Current Content-based Image Retrieval Methods,” IETE J. Res. 48(3\&4), pp. 261-271.

[5] Rui, Y. Huang, T. Ortega, M. Mehrotra, S.(1998) "Relevance Feedback: A Power Tool in Interactive Content-Based Image Retrieval," IEEE Transactions on Circuits and Systems for Video Technology, Vol. 8(5), pp. 644-655.

[6] Rui, Y., Huang, T.S., and Mehrotra,S.(1997) “Content-based Image Retrieval with Relevance Feedback in MARS," in Proc. IEEE Int. Conf. on Image proc.

[7] S. D. MacArthur, C. E. Brodley, and C. R. Shyu, (2000), "Relevance Feedback Decision Trees in Content-based Image Retrieval," in Proc. IEEE Work-shop Content-based Access of Image and Video Libraries, pp.68-72.

[8] I. J. Cox, M.L. Miller, T.P. Minka, T.V.Papathomas, and P.N.Yianilos,(2000), "The Bayesian Image Retieval System, PicHunter: Theory, Implementation and Psychophysical Experiments,” IEEE Tran. on Image Processing, Vol. 9,Issue 1, pp.20-37.

[9] Z. Su H. Zhang, S. Li, and S. Ma, (2003) "Relevance Feedback in Content-based Image Retrieval: Bayesian framework, Feature Subspaces, and Progressive Learning," IEEE Trans. Image Process. vol. 12, no. 8, pp. 924-936.

[10] Tong S. and Chang E., (2001), "Support Vector Machines Active Learning for Image Retrieval" Proc. ACM Multimedia.

[11] K. Tieu and P. Viola,(2003), "Boosting image retrieval," in Proc. IEEE Conf. Computer Vision Pattern Recognition, pp. 228-235.

[12] Zhou, X. S. and Huang, T. S.,(2003), "Relevance Feedback in image retrieval: A Comprehensive review," Multimedia systems, 8, 6, pp. 536-544.

[13] Zhi-Hua Zhou, Ke-Jia Chen, and Hong-Bin Dai,(2006), "Enhanced Relevance Feedback in Image Retrieval Using Unlabeled Data," ACM trans. on informations systems, vol.24, issue 2, pp. 219-244.

[14] Marin Ferecatu, Nozha Boujemaa, Michel Crucianu,(2008), "Semantic interactive image retrieval combining visual and conceptual content description," ACM multimedia systems Journal, vol. 13, No. 5-6, pp. 309-322. 
International Journal of Web \& Semantic Technology (IJWesT) Vol.2, No.4, October 2011

[15 Steven C. H. Hoi, Michael R. Lyu, and Rong Jin(2006), “A Unified Log-Based Relevance Feedback Scheme for Image Retrieval”, IEEE trans. on Knowledge and Data Engineering, vol. 18, no. 4.

[16] Tong, S., Chang, E. (2001), "Support vector machine active learning for image retrieval", In Proceedings of the 9th ACM international conference on Multimedia, pp. 107-118. ACM Press.

[17] Tong, S., Koller, D.(2000), "Support vector machine active learning with applications to text classification" In Proceedings of ICML-00, 17th International Conference on Machine Learning, pp. 999-1006. Morgan Kaufmann .

[18] Manesh Kokare, P.K. Biswas, and B.N. Chatterji,(2005) "Texture Image retrieval using New Rotated Complex Wavelet Filters," IEEE Trans. on systems, man, and Cybernetics-Part B: Cybernetics, vol. 35, no.6.

[19] Chiou-Ting Husu and Chuech-Yu Li,(2005), "Relevance Feedback Using Generalized Bayesian Framework with Region Based optimization Learning”, IEEE trans. on Image Processing, vol. 14, No.10.

[20] Anca Loredana Ion, Liana Stanescu, and Dan Burdescu,(2009), "Semantic Based Image Retrieval using Relevance Feedback", international Conference on Computer as a Tool, pp. 303-310, Warsaw

[21] N.G. Kingsbury, "Image processing with complex wavelet," Phil. Trans. Roy. Soc. London A, vol. 357, pp. 2543-2560 (1999).

[22] N. G. Kingsbury,(2001), "Complex wavelets for shift invariant analysis and filtering of signals," J.App. Comput. Harmon. Anal., vol. 10, no.3, pp.234-253.

[23] I. Selesnick, R. Baraniuk, and N. Kingsbury,(2005), "The dual-tree complex wavelet transform," IEEE Signal Process. Mag., vol.22, no. o6, pp.123-151.

[24] Y. Freund and R. E. Schapire,(1997), “A decision -theoretic generalization of online learning and an application to boosting”, J. Comput. Syst. Sci., 55(1):119-139.

[25] Rongtao Ding, Xinhao Ji, and Linting Zhu,(2007), "Research on the Relevance Feedback-based Image retrieval in Digital library”, PWASET vol. 25, ISSN 1307-6884. 